Series A

I. MATHEMATICA

474

\title{
A CAPACITY INEQUALITY FOR QUASIREGULAR MAPPINGS
}

BY

O. MARTIO

H E L S I N K I 1970

S U O M A L A I N E N T I DEAKA T EM I A

doi:10.5186/aasfm.1971.474 
Communicated 13 March 1970 by OLLI Lehto 


\section{Introduction}

In [7] two capacity inequalities for quasiregular mappings are introduced. The first is the outer dilatation capacity inequality

$$
\frac{1}{K_{o}(f) N(f, A)} \operatorname{cap} E \leqq \operatorname{cap} f E
$$

where $E=(A, C)$ is a normal condenser in the domain $G$ of a nonconstant quasiregular mapping $f$ with an outer dilatation $K_{o}(f)$ and $N(f, A)=\sup$ card $\left(f^{-1} f(x) \cap A\right)$ over $x \in A$. The second inequality is the inner dilatation $K_{I}(f)$ capacity inequality

$$
\operatorname{cap} f E \leqq K_{I}(f) \operatorname{cap} E
$$

where $E$ is any condenser in $G$. In this paper we show that it is possible, under certain assumptions, to divide $K_{I}(f)$ in (1.2) by $N(f, A)$ corresponding to the situation in (1.1). We also give applications of this theorem.

The main results of this paper were presented in the Roumanian-Finnish seminar on Teichmüller spaces and quasiconformal mappings at Brasov $25-30.8 .1969$ (see [8]).

\section{Notation and terminology}

We shall use the same notation and terminology as in $[7,2.1$, p. 5] with a few exceptions and additional concepts.

For $A \subset R^{n}$ we let $\operatorname{dim} A$ denote the topological dimension of $A$ (see $[6])$.

By $[a, b], a, b \in R^{n}$, we mean the closed line segment $\{t a+(1-t) b$ $0 \leqq t \leqq 1\}$ in $R^{n}$. If $[a, b] \subset R^{1}$, then we suppose $b>a$, and $(a, b)$ denotes the corresponding open segment, the meanings of $[a, b)$ and $(a, b]$ being obvious. A path $\alpha:[a, b] \rightarrow R^{n},[a, b] \subset R^{1}$, is a continuous mapping. A curve is an injective path. We let $|\alpha|=\alpha([a, b])$. If $x, y \in A \subset R^{n}$ and $\alpha:[a, b] \rightarrow A$ is a path such that $\alpha(a)=x$ and $\alpha(b)=y$, then $\alpha$ is said to join $x$ and $y$.

A continuum in $R^{n}$ is a compact connected non-empty set which is not a point. 


\section{Lemmas on discrete, open, and sense-preserving mappings}

Suppose that $f: G \rightarrow R^{n}$ is discrete, open, and sense-preserving. At first we recall some notation and results in [7].

A domain $D$ is called a normal domain of $f$ if $\bar{D}$ is compact in $G$ and $\partial f D=f \partial D$. A normal neighbourhood of a point $x \in G$ is a normal domain $D$ of $f$ such that $D \cap f^{-1} f(x)=\{x\}$. If $r>0$ and $x \in G$, then we denote by $U(x, f, r)$ the $x$-component of $f^{-1} B^{n}(f(x), r)$. We frequently use the following lemma.

3.1. Lemma. [7, Lemma 2.9, p. 9] If $\bar{U}(x, f, r)$ is compact in $G$, then $U(x, f, r)$ is a normal domain of $f$. Furthermore, for every $x \in G$ there exists $\sigma_{x}>0$ such that for $0<r \leqq \sigma_{x}$

(1) $U(x, f, r)$ is a normal neighbourhood of $x$.

(2) $U(x, f, r)=U\left(x, f, \sigma_{x}\right) \cap f^{-1} B^{n}(f(x), r)$.

(3) $\bar{U}(x, f, r)=\bar{U}\left(x, f, \sigma_{x}\right) \cap f^{-1} \bar{B}^{n}(f(x), r)$.

(4) $d(U(x, f, r)) \rightarrow 0$ as $r \rightarrow 0$.

3.2. Lemma. $\operatorname{dim} B_{f}=\operatorname{dim} f B_{f} \leqq n-2$.

The important inequality $\operatorname{dim} B_{f} \leqq n-2$ is due to Černavskii $[1,2]$ (for a simpler proof see [13]). The equality $\operatorname{dim} B_{f}=\operatorname{dim} f B_{f}$ follows from $[3$, Theorem 2.2, p. 530].

3.3. Remark. If $A$ is a subset of $R^{n}$, then the inequality $\operatorname{dim} A \leqq n-2$ implies: $A$ does not disconnect any domain in $R^{n}$ [6, Corollary 1, p. 48].

If $D$ is a normal domain of $f$, then the topological degree $\mu(y, f, D)$ of $f$ is independent of $y \in f D$. This number is denoted by $\mu(f, D)$. For the next lemma we recall that $i(x, f)$ is the local topological degree of $f$ at $x \in G$ and $N(f, D)=\sup N(y, f, D)$ over $y \in R^{n}$ where $N(y, f, D)=$ card $\left(f^{-1}(y) \cap D\right)$.

3.4. Lemma. [7, Lemma 2.12, p. 11] If $D$ is a normal domain of $f$, then $\mu(f, D)=N(f, D)$. Furthermore, if $D$ is a normal neighbourhood of $x \in G$, then $i(x, f)=\mu(f, D)=N(f, D)$.

From the second assertion in Lemma 3.4 we conclude that $x \in B_{f}$ if and only if $i(x, f) \geqq 2$.

If $C$ is a non-empty and compact subset of $G$ and $y \in f C$, then we set

$$
M(y, f, C)=\sum_{x \in f^{-1}(y) \cap C} i(x, f) .
$$


The sum in (3.5) is finite since $f$ is discrete and $C$ compact in $G$. The number $M(f, C)=\inf M(y, f, C)$ over $y \in f C$ is called the minimal multiplicity of $f$ on $C$.

3.6. Lemma. Suppose that $A$ is open and $C \subset A$ non-empty and compact in $G$. Then $N(f, A) \geqq M(f, C)$.

Proof. Let $y \in f C$ and denote $\left\{x_{1}, x_{2}, \ldots, x_{k}\right\}=f^{-1}(y) \cap C . \quad B y$ Lemma 3.1 there exists $r>0$ such that $U_{i}=U\left(x_{i}, f, r\right)$ is a normal neighbourhood of $x_{i}$ and $U_{i} \subset A, 1 \leqq i \leqq k$. By Lemma 3.2 there exists $y_{0} \in B^{n}(y, r) \backslash f B_{f}$. Lemma 3.4 implies

$$
N(f, A) \geqq N\left(y_{0}, f, A\right) \geqq \sum_{i=1}^{k} N\left(y_{0}, f, l_{i}^{*}\right)=\sum_{i=1}^{k} i\left(x_{i}, f\right) \geqq M(f, C),
$$

and the lemma follows.

3.7. Lemma. Suppose that $D$ is a normal domain of $f$. Then $M(f, \bar{D})=\mu(f, D)$.

Proof. Clearly $M(f, \bar{D}) \leqq \mu(f, D)$. Let $y \in f \bar{D}$ be such that $M(f, \bar{D})=$ $M(y, f, \bar{D})$. Choose a neighbourhood $U$ of $\bar{D}$ such that $f^{-1}(y) \cap \bar{U} \backslash \bar{D}=$ $O$, and let $r<d(y, f \hat{c} U)$ be such that the sets $U_{i}=U\left(x_{i}, f, r\right)$ are normal neighbourhoods of the points $x_{1}, x_{2}, \ldots, x_{k}$ of $f^{-1}(y) \cap \bar{D}$. By Lemma 3.2 there exists a point $y_{0} \in B^{n}(y, r) \cap f D \backslash f B_{f}$. Lemma 3.4 yields

$$
M(f, \bar{D})=\sum_{i=1}^{k} i\left(x_{i}, f\right)=\sum_{i=1}^{k} \mu\left(f, C_{i}\right) \geqq \operatorname{card}\left(f^{-1}\left(y_{0}\right) \cap D\right)=\mu(f, D) .
$$

The lemma follows.

In view of Remark 3.3 the proof of the following lemma is clear.

3.8. Lemma. Suppose that $D \subset R^{n}$ is a domain. $x$ and y are distinct points in $D$, and $A \subset R^{n}$ is a closed sei such that dim $A \leq n-2$. Then there is a curve $x:[a, b] \rightarrow I)$ which joins $x$ and $y$, and such that $\alpha((a, b)) \cap A=\varnothing$.

\section{Path joinable points}

Suppose that $f: G \rightarrow R^{n}$ is discrete, open, and sense-preserving. Let $D \subset G$ be a domain, $y$ and $\bar{y}$ points in $f D$, and $\beta:[a, b] \rightarrow f D$ a path which joins $y$ and $\bar{y}$. We say that $x \in f^{-1}(y) \cap D$ and $\bar{x} \in f^{-1}(\bar{y}) \cap D$ are $(f, D, \beta)$-joinable points if there exists a path $x:[a, b] \rightarrow D$ such that $f \circ \alpha=\beta$ and $\alpha$ joins $x$ and $\bar{x}$. 
4.1. Lemma. [7, Lemma 2.7, p. 9] Suppose that $D$ is a normal domain of $f, \beta:[a, b] \rightarrow f D$ is a path, $a \leqq t_{0} \leqq b$, and $x_{0} \in D$ such that $\beta\left(t_{0}\right)=$ $f\left(x_{0}\right)$. Then there is a path $\alpha:[a, b] \rightarrow D$ such that $\alpha\left(t_{0}\right)=x_{0}$ and $f \circ \alpha=\beta$.

The path $\alpha$ is called a lift of $\beta$. We need the above lemma only for curves in which case it is essentially due to Whyburn [15, (2.1), p. 186]. Observe that if $\beta$ is a curve then its lift $\alpha$ is a curve.

4.2. Here we study the case where $y$ and $\bar{y}, y \neq \bar{y}$, belong to the image of some normal domain $D$ of $f$ and $\beta:[a, b] \rightarrow f D$ is a straight line segment joining $y$ and $\bar{y}$. These are kept fixed in the following discussion.

Let

$$
\begin{aligned}
& f^{-1}(y) \cap D=\left\{x_{1}, x_{2}, \ldots, x_{s}\right\}, \\
& f^{-1}(\bar{y}) \cap D=\left\{\bar{x}_{1}, \bar{x}_{2}, \ldots, \bar{x}_{\bar{s}}\right\}
\end{aligned}
$$

where each point is counted according to its local topological degree. Then $s=\mu(f, D)=\bar{s}$. Denote $S=\{1,2, \ldots s\}$.

4.3. Lemma. There is a bijection $q: S \rightarrow S$ such that if $i \in S$ then $x_{i}$ and $\bar{x}_{q(i)}$ are $(f, D, \beta)$-joinable point.

Proof. By Lemma 3.1 it is possible to cover $\beta$ by balls $B^{n}\left(y_{k}, r_{k}\right) \subset f D$, $k=1,2, \ldots, m, m>2$, such that (1) $y_{k} \in|\beta|,(2) \quad y=y_{1}, \bar{y}=y_{m}$, (3) $\left|y-y_{2}\right|<\left|y-y_{3}\right|<\ldots<\left|y-y_{m}\right|$, (4) $B^{n}\left(x_{k}, r_{k}\right) \cap B^{n}\left(x_{k+1}, r_{k+1}\right) \neq$ O, $\quad 1 \leqq k \leqq m-1$, and $(5)$ if $x \in f^{-1}\left(y_{k}\right) \cap D$ then $U\left(x, f, r_{k}\right)$ is a normal neighbourhood of $x$. Let $V=\cup B^{n}\left(y_{k}, r_{k}\right)$.

Lemmas 3.2 and 3.8 show that we can construct a curve $\gamma:[a, b] \rightarrow V$ such that it joins $y$ and $\bar{y} \cdot \gamma((a, b)) \cap f\left(B_{f} \cap D\right)=\varnothing$, and $\gamma^{\prime} \cap B^{n}\left(y_{k}, r_{k}\right)$ is connected for every $k, 1 \leqq k \leqq m$. Let $z \in B^{n}\left(y, r_{1}\right) \cap|\gamma| \backslash\{y\}$ and $\bar{z} \in B^{n}\left(\bar{y}, r_{m}\right) \cap|\gamma| \backslash\{\bar{y}\}$. Then the local topological index at each point of $f^{-1}(z) \cap D$ or $f^{-1}(\bar{z}) \cap D$ is one. hence. by Lemma 3.4, we may write

$$
\begin{aligned}
& f^{-1}(z) \cap D=\left\{a_{1} \cdot a_{2} \ldots . a_{s}\right\} . \\
& f^{-1}(\bar{z}) \cap D=\left\{\bar{a}_{1} \cdot \bar{a}_{2} \ldots . \bar{a}_{s}\right\}
\end{aligned}
$$

where all the points $a_{i}$ and $\bar{a}_{i}, \quad 1 \leqq i \leqq s$. are distinct.

Define the mapping $q: S \rightarrow S$ as follows: If $i \in S$ then $a_{i} \in f^{-1}(z) \cap D$. By Lemma 4.1 we lift the curve $\gamma$ to a curve $\gamma^{\prime}:[a, b] \rightarrow D$ such that $a_{i} \in\left|\gamma^{\prime}\right|$ and $f \circ \gamma^{\prime}=\gamma$. Then some $\bar{a}_{j} \in f^{-1}(\bar{z}) \cap D$ belongs to $\left|\gamma^{\prime}\right|$. Since $i(x, f)=1$ for $x \in \gamma^{\prime}((a, b))$, there is only one lift $\gamma^{\prime}$ of $\gamma$ such that $a_{i} \in\left|\gamma^{\prime}\right|$. Thus $\bar{a}_{j}$ is uniquely determined. Set $\varphi(i)=j$.

Finally we construct the required lift for the segment $\beta$. Let $\gamma, i \in S$, 
and $\gamma^{\prime}$ be as above. For each $k, 1 \leqq k \leqq m$, let $U_{k}=U\left(x_{0}^{k}, f, r_{k}\right)$ be the only normal neighbourhood of the normal neighbourhoods $U\left(x, f, r_{k}\right)$. $x \in f^{-1}\left(y_{k}\right) \cap D$, which meets $\gamma^{\prime}$. For if there exist two such normal neighbourhoods, say $U_{k}$ and $U_{k}^{\prime}$, then, since $|\gamma| \cap B^{n}\left(y_{k}, r_{k}\right)$ is connected, we obtain

$$
f\left(\mid \gamma^{\prime} \cap U_{k}\right)=\gamma \cap \cap B^{n}\left(y_{k}, r_{k}\right)=f\left(\left|\gamma^{\prime}\right| \cap U_{k}^{\prime}\right)
$$

which is impossible. Let $W=\cup \mathcal{C}_{k}$. The set $W$ is a domain which contains $\left|\gamma^{\prime}\right|$ and the points $x_{i}$ and $\bar{x}_{q(i)}$. Pick points $y_{k}^{\prime} \in B^{n}\left(y_{k}, r_{k}\right) \cap B^{n}\left(y_{k+1}, r_{k+1}\right)$. $k=1,2, \ldots, m-1$, so that they lie between $y_{k}$ and $y_{k+1}$ on $\beta$. Divide the segment $\beta$ into non-overlapping segments $\left|\beta_{1}^{\prime}\right|==\left[y, y_{1}^{\prime}\right]$, $\left|\beta_{2}\right|=\left[y_{1}^{\prime}, y_{2}\right],\left|\beta_{2}^{\prime}\right|=\left[y_{2}, y_{2}^{\prime}\right], \ldots,\left|\beta_{m}\right|=\left[y_{m-1}^{\prime}, \bar{y}\right]$. Let $x_{k}^{\prime} \in f^{-1}\left(y_{k}^{\prime}\right) \cap L_{k}^{\prime} \cap$ $U_{k+1}$ and suppose that $\alpha_{k}:[a, b] \rightarrow U_{k}$ (resp. $\left.\alpha_{k}^{\prime}:[a, b] \rightarrow U_{k}\right)$ is a lift of $\beta_{k}$ (resp. $\beta_{k}^{\prime}$ ) such that $x_{k}(a)=x_{k-1}^{\prime}$ for $2 \leqq k \leqq m$ (resp. $\alpha_{k}^{\prime}(b)=x_{k}^{\prime}$ for $1 \leqq k \leqq m-1)$. Since $f^{-1}\left(y_{i}\right) \cap C_{k}=\left\{x_{0}^{k}\right\}, \quad x_{k}(b)=x_{k}^{\prime}(a)=x_{0}^{i_{k}} \quad$ for $2 \leqq k \leqq m-1$ and $x_{1}^{\prime}(a)=x_{i},>_{m}(b)=\bar{x}_{t_{f}(i)}$. Joining these curves, in the order $\alpha_{1}^{\prime}, \alpha_{2}, \alpha_{2}, \ldots, x_{m}$, into one single curve and performing an obvious change of the parameter we obtain the required lift of $\beta$. The lemma follows.

\section{Capacity inequality}

Suppose that $f: G-R^{n}$ is a non-constant quasiregular mapping. From Rešetnjak's results $[9,10], f$ is discrete, open, and sense-preserving, hence we may use the results obtained in the sections 3 and 4 .

Following [7, 5.2, p. 24] we call a pair $E=(A, C)$ a condenser if $A \subset R^{n}$ is open and $C$ is non-empty and compact in $A$. A condenser $E$ is said to be in $G$ if $A \subset G . E$ is a normal condenser if $A$ is a normal domain of $f$. The image of a condenser $E$ in $G$ is a condenser $f E=$ $(f A, f C)$. The capacity of $E$ is defined as

$$
\operatorname{cap} E=\operatorname{cap}(A, C)=\inf _{u \in W_{0}(E)} \int_{-1} \tau^{n} d m
$$

where $W_{0}(E)=W_{0}(A, C)$ is the set of all non-negative real-valued functions $u \in C_{0}(A)$ such that $u$ is $A C L$ and $u \mid C \geqq 1$. It is not difficult to show (see $[7$, Lemma 5.5, p. 25]) that

$$
\operatorname{cap} E=\inf _{u \in W_{0}^{\prime}(E)}|\nabla u|_{A}^{n} d m
$$

where $W_{0}^{\infty}(E)=W_{0}(E) \cap C_{0}^{\infty}(A)$. 
5.1. Theorem. Suppose that $f: G \rightarrow R^{n}$ is a non-constant quasiregular mapping. If $E=(A, C)$ is a condenser in $G$ and $N(f, A)<\infty$, then

$$
\operatorname{cap} f E \leqq \frac{K_{I}(f) N(f, A)^{n-1}}{M(f, C)^{n}} \operatorname{cap} E .
$$

The proof of this result is closely related to that of the formula (1.2) [7, Theorem 7.1, p. 29]. At first we prove two lemmas.

Suppose that $u \in W_{0}^{\infty}(E)$. Define $v: f A \rightarrow R^{1}$ by

$$
v(y)=\frac{1}{M(f, C)} \sum_{x \in f=i(y)} i(x, f) u(x) .
$$

The sum in (5.3) contains only a finite number of non-zero terms since $f$ is discrete and spt $u$ is compact in $G$.

\subsection{Lemma. The function $v$ has the properties}

(1) $\operatorname{spt} v$ is compact in $f A$, moreover $f($ spt $u)=\operatorname{spt} v$.

(2) $v(y) \geqq 1, y \in f C$.

(3) $v$ is continuous.

Proof. Set $U=\{x \in A: u(x) \neq 0\}$ and $V=\{x \in f A, v(x) \neq 0\}$. Then $f U=V$. By the continuity of $f, f(\operatorname{spt} u)=f \bar{C}=\bar{V}=\operatorname{spt} v$. This implies that spt $v$ is compact in $f A$.

To prove (2) let $y \in f C$ and denote $\left\{x_{1}^{2}, x_{2}, \ldots, x_{k}\right\}=C \cap f^{-1}(y)$. Then

$$
\begin{aligned}
v(y)=\frac{1}{M(f, C)} \sum_{x \in f^{-1}(,)} i(x, f) u(x) \geqq \frac{1}{M\left(f, C^{\prime}\right)} \sum_{i=1}^{k} i\left(x_{i}, f\right) u\left(x_{i}\right) & \\
& \geqq \frac{1}{M(f, C)} \sum_{i=1}^{k} i\left(x_{i}, f\right) \geqq 1,
\end{aligned}
$$

since every $u\left(x_{i}\right) \geqq 1$.

For (3), let $y \in f A$ and $\varepsilon>0$. We may suppose that $y \in \operatorname{spt} v$ since otherwise $(3)$ is trivial. Choose a neighbourhood $C$ of spt $u$ such that $\bar{C}$ is compact in $A$ and $y \notin f \partial U$. Let $\left\{x_{1}, x_{2}, \ldots, x_{k}\right\}=f^{-1}(y) \cap U$. By Lemma 3.1 and the continuity of $u$, there exists a number $r$ such that $0<r<d(y, f \partial U)$ and the normal neighbourhoods $U_{i}=C^{r}\left(x_{i}, f, r\right)$, $1 \leqq i \leqq k$, satisfy the conditions: (i) $\bar{U}_{i} \subset U$ and (ii) ' $u(x)-u\left(x_{i}\right)<\varepsilon$, $x \in U_{i}$. Denote $U_{0}=\cup U_{i}$. Let $z \in B^{n}(y, r)$. Then $f^{-1}(z) \cap U \subset U_{0}$. Fix $i, \quad 1 \leqq i \leqq k$, and let $f^{-1}(z) \cap U_{i}=\left\{z_{1}^{i}, z_{2}^{i}, \ldots, z_{l(i)}^{i}\right\}$.

Since $f$ is discrete, there exists $C_{1}>0$ such that

$$
\sum_{x \in f^{-1}\left(z_{1}\right) \cap U_{n}} i\left(x, f^{\prime}\right) \leqq C_{1}
$$


for all $z_{1} \in R^{n}$. Because $U_{i}$ is a normal neighbourhood of $x_{i}$, we obtain

$$
i\left(x_{i}, f\right)=\mu\left(f, U_{i}\right)=\sum_{j=1}^{l(i)} i\left(z_{j}^{i}, f\right) .
$$

This yields

$$
\begin{aligned}
\mid i\left(x_{i}, f\right) u\left(x_{i}\right)- & \sum_{j=1}^{l(i)} i\left(z_{j}^{i}, f\right) u\left(z_{j}^{i}\right)|=| \sum_{j=1}^{l(i)} i\left(z_{j}^{i}, f\right) u\left(x_{i}\right)-\sum_{j=1}^{l(i)} i\left(z_{j}^{i}, f\right) u\left(z_{j}^{i}\right) \mid \\
& \leqq \sum_{j=1}^{l(i)} i\left(z_{j}^{i}, f\right)\left|u\left(x_{i}\right)-u\left(z_{j}^{i}\right)\right|<C_{1} \varepsilon .
\end{aligned}
$$

Stmming these inequalities over $i$ gives

$$
\begin{gathered}
\mid v(y)-v(z)=\frac{1}{M(f, C)} \sum_{i=1}^{k}\left[i\left(x_{i}, f\right) u\left(x_{i}\right)-\sum_{j=1}^{l(i)} i\left(z_{j}^{i}, f\right) u\left(z_{j}^{i}\right)\right] \\
<\frac{k C_{1}}{M(f, C)} \varepsilon \leqq \frac{C_{1}^{2}}{M(f, C)} \varepsilon .
\end{gathered}
$$

The inequality holds for every $z \in B^{n}(y, r)$, hence the lemma is proved.

\subsection{Lemma. $v$ is $A C L$.}

Proof. It is enough to prove that $v$ is $A C L$ in a neighbourhood of each point of spt $v$. Fix $y_{0} \in \operatorname{spt} v$ and let $f^{-1}\left(y_{0}\right) \cap \operatorname{spt} u=\left\{x_{1}, x_{2}, \ldots, x_{q}\right\}$. Choose $r_{0}$ such that $0<r_{0}<d\left(y_{0}, \partial f A\right)$ and such that the domains $U\left(x_{j}, f, r_{0}\right)$ are normal neighbourhoods of $x_{j}$. Next choose a positive number $r_{1} \leqq r_{0}$ such that

$$
B^{n}\left(y_{0}, r_{1}\right) \cap f\left(\operatorname{spt} u \backslash \bigcup_{j=1}^{q} U\left(x_{j}, f, r_{0}\right)\right)=\varnothing .
$$

Then the components of $f^{-1} B^{n}\left(y_{0}, r_{1}\right)$ which meet spt $u$ are the sets $U_{j}=U\left(x_{j}, f, r_{1}\right)$. Set $U=U U_{j}$. We have

$$
v(y)=\frac{1}{M(f, C)} \sum_{x \in f^{-1}(y) \cap L^{-}} i(x, f) u(x)
$$

for every $y \in B^{n}\left(y_{0}, r_{1}\right)$.

Let $Q$ be an open $n$-interval with closure in $B^{n}\left(y_{0}, r_{1}\right)$. Write $Q=Q_{0} \times J$ where $Q_{0}$ is an $(n-1)$-interval in $R^{n-1}$ and $J$ is an open segment in some $x_{i}$-axis, $1 \leqq i \leqq n$. For each Borel set $F \subset Q_{0}$ put $\psi(F)=m\left(U \cap f^{-1}(F \times J)\right)$. Then $\psi$ is a completely additive set function in the family of all Borel sets in $Q_{0}$. By Legesgue's theorem $\bar{\psi}^{\prime}(z)<\infty$ for almost all $z \in Q_{0}$. Fix such $z$ and set $J_{z}=\{z\} \times J$. It is sufficient to show that $v$ is absolutely continuous on $\bar{J}_{z}$. 
Let $\Phi$ denote the set of all continuous mappings $g: \bar{J}_{\tilde{z}} \rightarrow U$ such that $f \circ g$ is the identity mapping of $\bar{J}_{z}$. Observe that every $g \bar{J}_{z}$ is contained in some $U_{j}$.

Let $\left[y_{1}, \bar{y}_{J^{\prime}}\right],\left[y_{2}, \bar{y}_{2}\right], \ldots,\left[y_{p}, \bar{y}_{p}\right]$ be a collection of disjoint closed segments on $\bar{J}_{z}$. Fix $r, 1 \leqq r \leqq p$, and $j, 1 \leqq j \leqq q$. Using the notation of 4.2 with $D=U_{j}, \quad y=y_{r}, \quad \bar{y}=\bar{y}_{r}, \quad|\beta|=\left[y_{r}, \bar{y}_{r}\right]$, and

$$
\begin{aligned}
& f^{-1}\left(y_{r}\right) \cap U_{j}=\left\{x_{i}^{r j} \mid i=1,2, \ldots, s(j)\right\}, \\
& f^{-1}\left(\bar{y}_{r}\right) \cap U_{j}=\left\{\bar{x}_{i}^{r j} \mid i=1,2, \ldots, s(j)\right\}
\end{aligned}
$$

where each point is counted according to its local topological degree, we obtain

$$
\sum_{x \in f^{-1}\left(y_{r}\right) \cap U_{j}} i(x, f) u(x)-\sum_{x \in f^{-1}\left(\bar{y}_{r}\right) \cap U_{j}^{-}} i(x, f) u(x)=\sum_{i=1}^{s(j)}\left(u\left(x_{i}^{r j}\right)-u\left(\bar{x}_{r_{f}(i)}^{r j}\right)\right)
$$

where $\varphi=\varphi_{r j}$ is the mapping given by Lemma 4.3. Since $x_{i}^{r j}$ and $\bar{x}_{r(i)}^{r j}$ are $\left(f, U_{j}, \beta\right)$-joinable points, there exists $g_{i}^{r j} \in \Phi$ such that $g_{i}^{r j}\left(y_{r}\right)=x_{i}^{r j}$ and $g_{i}^{r j}\left(\bar{y}_{r}\right)=\bar{x}_{q(i)}^{r j}$. Summing over $j$ and $r$ in (5.7) we have by (5.6)

$$
\sum_{r=1}^{p}\left|v\left(y_{r}\right)-v\left(\bar{y}_{r}\right)\right| \leqq \sum_{r=i}^{p} \sum_{j=1}^{q} \sum_{i=1}^{s(j)}\left|u\left(g_{i}^{r j}\left(y_{r}\right)\right)-u\left(g_{i}^{r j}\left(\bar{y}_{r}\right)\right)\right| .
$$

Since $u \in C_{0}^{\infty}(A)$ there is a constant $C_{1}$ such that $u(x)-u(y) \leqq$ $C_{1}|x-y|$ for all $x$ and $y$ in $U$. Then (5.8) implies

$$
\sum_{r=1}^{p}\left|v\left(y_{r}\right)-v\left(\bar{y}_{r}\right)\right| \leqq C_{1} \sum_{j=1}^{q} \sum_{i=1}^{s(j)} \sum_{r=1}^{p}\left|g_{i}^{r j}\left(y_{r}\right)-g_{i}^{r j}\left(\bar{y}_{r}\right)\right| .
$$

By [7, Lemma 7.10, p. 33] for every $\varepsilon>0$ there exists $\delta>0$ such that for each $j, 1 \leqq j \leqq q$, and $i, 1 \leqq i \leqq s(j)$,

$$
\sum_{r=1}^{p}\left|g_{i}^{r j}\left(y_{r}\right)-g_{i}^{r_{j}}\left(\bar{y}_{r}\right)\right|<\varepsilon / q s(j) C_{1}
$$

if $\sum\left|y_{r}-\bar{y}_{r}\right|<\delta$. Hence the left side of (5.9) is smaller than $\varepsilon$ if $\sum\left|y_{r}-\bar{y}_{r}\right|<\delta$. The lemma is proved.

5.10. Proof for Theorem 5.1. Lemmas 5.4 and 5.5 show that for each $u \in W_{0}^{\infty}(E) \quad v$ belongs to $W_{0}(f E)$. Let $y_{0} \in \operatorname{spt} v \backslash f\left(\mathrm{spt} u \cap B_{f}\right)$. By the same method as in $[7$, Lemma 7.15, p. 35] we can find a neighbourhood $V_{0}$ of $y_{0}$ such that for every connected neighbourhood $V \subset V_{0}$ of $y_{0}$ the following conditions hold:

(1) $V \cap f\left(\right.$ spt $\left.u \cap B_{f}\right)=\varnothing$.

(2) The components of $f^{-1} V$ which meet spt $u$ form a finite collection $D_{1}, D_{2}, \ldots, D_{k}$. 
(3) $f$ defines a homeomorphism $f_{i}: D_{i} \rightarrow V$, i.e. $f_{i}=f \quad D_{i}$ is a quasiconformal mapping, $1 \leqq i \leqq k$.

Furthermore we may suppose that

(4) $d\left(D_{i}\right)<d(\operatorname{spt} u, \partial A), \quad 1 \leqq i \leqq k$.

Set $g_{i}=f_{i}^{-1}$. Since $i(x, f)=1$ for $x \in G \backslash B_{f}$,

$$
v(y)=\frac{1}{M(f, C)} \sum_{i=1}^{k} u\left(g_{i}(y)\right)
$$

for all $y \in V$. Thus

$$
|\nabla v(y)| \leqq \frac{1}{M(f, C)} \sum_{i=1}^{l_{k}}\left|\nabla u\left(g_{i}(y)\right)\right|\left|g_{i}^{\prime}(y)\right|
$$

a.e. in $V$ because every $g_{i}$ is a.e. differentiable in $V$.

There is a countable number of open disjoint cubes $Q_{1}, Q_{2}, \ldots$ such that $f A \backslash f\left(\operatorname{spt} u \cap B_{f}\right)=\cup \bar{Q}_{j}$ and such that if $Q_{j}$ meets spt $v$ then the conditions (1)-(4) are satisfied with $V=Q_{j}$. By [7, Lemma 2.27, p. 15] $m\left(f B_{f}\right)=0$, hence

$$
\operatorname{cap} f E \leqq \sum_{j=i}^{\infty} \int_{Q_{j}} \mid \nabla v^{n} d m
$$

Fix $Q_{j}$. If it does not meet spt $v$, then

$$
\int_{Q_{j}} \nabla v{ }^{n} d m=0 .
$$

If $Q_{j}$ meets spt $v$, let $g_{i}: Q_{j} \rightarrow D_{i}, \quad l \leqq i \leqq k$, denote the inverse mappings given by (3). Minkowski's inequality yields by (5.11)

$$
\begin{aligned}
\left(\int_{Q_{j}}|\nabla v|^{n} d m\right)^{1 / n} & \leqq \frac{1}{M(f, C)} \sum_{i=1}^{k}\left(\int_{Q_{j}}\left|\nabla u\left(g_{i}(y)\right)^{n} g_{i}^{\prime}(y)\right|^{n} d m(y)\right)^{1 / n} \\
& \leqq \frac{K_{I}(f)^{1 / n}}{M(f, C)} \sum_{i=1}^{k}\left(\int_{g_{i} Q_{j}} \mid \nabla u^{i n} d m\right)^{1 / n}
\end{aligned}
$$

where we have used the quasiconformality of $g_{i}$ 's in the last step. It follows from (4) that $k \leqq N(f, A)$, hence Hölder's inequality gives

$$
\begin{aligned}
\left(\int_{Q_{j}}|\nabla v|^{n} d m\right)^{1 / n} & \leqq \frac{K_{I}(f)^{1 / n} k^{1-1 / n}}{M(f, C)}\left(\sum_{i=1}^{k} \int_{g_{i} Q_{j}} \mid \nabla u^{n} d m\right)^{1 / n} \\
& \leqq \frac{K_{I}(f)^{1 / n} N(f, A)^{1-1 / n}}{M(f, C)}\left(\int_{{ }^{-1} Q_{j}} \nabla u^{\prime n} d m\right)^{1 / n}
\end{aligned}
$$


By (5.12)

$$
\begin{aligned}
\operatorname{cap} f E & \leqq \frac{K_{I}(f) N(f, A)^{n-1}}{M(f, C)^{n}} \sum_{j=1}^{\infty} \int_{f^{-1} Q_{j}}|\nabla u|^{n} d m \\
& \leqq \frac{K_{I}(f) N(f, A)^{n-1}}{M(f, C)^{n}} \int_{A}|\nabla u|^{n} d m
\end{aligned}
$$

This holds for every $u \in T_{0}^{\infty}(E)$, hence the formula (5.2) is proved.

It is easy to give an example of a quasiregular mapping $f: G \rightarrow R^{n}$ and a condenser $E=(A, C)$ in $G$ such that $N(f, A)^{n-1} / M(f, C)^{n}>1$. For example. consider the analytic function $f: R^{2} \rightarrow R^{2}, z \mapsto z^{2}$, and the condenser $(A, C)=\left(B^{2},\{1 / 2\}\right)$, both given in complex notation. Then $N(f, A) / M(f, C)^{2}=2 / 1=2>1$. Hence the inequality (5.2) may be worse than (1.2). We also remark that by Lemma 3.6 the inequality $N(f, A) \geqq M(f, C)$ is satisfied for any condenser $(A, C)$ in $G$. However. in some important cases the condition of the following corollary holds.

5.13. Corollary. Suppose that $f: G \rightarrow R^{n}$ is a non-constant quasiregular mapping and $E=(A, C)$ a condenser in $G$ such that $N(f, A)=M(f, C)$. Then

$$
\text { cap } f E \leqq \frac{K_{I}(f)}{N(f, A)} \operatorname{cap} E .
$$

The next corollary gives a rery precise estimate for the variation of the caproity.

5.14. Corollary. Suppose that $f: G \rightarrow R^{n}$ is a non-constant quasiregular mapping and $E=(A, C)$ a normal condenser in $G$ such that $N(f, A)=$ $M(f, C)$. Then

$$
\frac{1}{K_{0}(f) N(f, A)} \operatorname{cap} E \leqq \operatorname{cap} f E \leqq \frac{K_{I}(f)}{\Gamma(f, A)} \operatorname{cap} E .
$$

This follows immediately from (1.1) and Corollary 5.13.

5.15. Remark. We give an example of the case $N(f . A)=M\left(f, C^{\prime}\right)$. Let $f: G \rightarrow R^{n}$ be discrete, open, and sense-preserring. Suppose that $A=U\left(x, f, r_{1}\right)$ is a normal neighbourhood of $x \in G$. Let $0<r<r_{1}$ and denote $C=\bar{U}(x, f, r)$. Then $E=(A, C)$ is a condenser in $G$. and by Lemmas 3.4 and 3.7.

$$
N(f, A)=i(x, f)=\mu(f, A)=M(f, C) .
$$




\section{Applications}

The first theorem generalizes the well-known fact: If $f: G \rightarrow R^{2}$ is an analytic function, then $f^{\prime}(x)=0$ for $x \in B_{f}$.

6.1. Theorem. Suppose that $f: G \rightarrow R^{n}$ is quasiregular and $x \in G$. If $K_{I}(f)<i(x, f)$, then $f$ is differentiable at $x$ and $f^{\prime}(x)=0$.

Proof. We may suppose that $f$ is not a constant. Let $U_{0}=U\left(x, f, r_{0}\right)$ be a normal neighbourhood of $x \in G$. Suppose that $r_{1}>0$ is so small that $U_{0} \supset \bar{B}^{n}\left(x, r_{1}\right)$. By Lemma 3.1 there exists a positive number $r_{2}$ such that for $0<r \leqq r_{2} \quad U(x, f, r) \subset B^{n}\left(x, r_{1}\right)$. Let $y \in U\left(x, f, r_{2}\right)$ and suppose $y \neq x$. Set $r=|f(x)-f(y)|>0$. Define a condenser $E$ in $G$ as $\left(U_{0}, \bar{U}(x, f, r)\right)$. By Remark 5.15 we have

$$
i(x, f)=M(f, \bar{U}(x, f, r))=N\left(f, U_{0}\right) .
$$

Suppose that the condition $K_{I}(f)<i(x, f)$ is satisfied. By Corollary 5.13

$$
\frac{\omega_{n-1}}{\left(\log \left(r_{0} / r\right)\right)^{n-1}}=\operatorname{cap} f E \leqq \frac{K_{I}(f)}{i(x, f)} \operatorname{cap} E=\frac{1}{\alpha} \operatorname{cap} E
$$

where $\alpha>1$. Let

$$
\begin{aligned}
& L^{*}(r)=\inf \left\{\beta>0 \mid B^{n}(x, \beta) \supset U(x, f, r)\right\}, \\
& l^{*}(r)=\sup \left\{\beta>0: B^{n}(x, \beta) \subset U(x, f, r)\right\}
\end{aligned}
$$

(sce $\left[7,4.1\right.$, p. 17]). Since the condenser $\left(B^{n}\left(x, r_{1}\right), \bar{B}^{n}\left(x, L^{*}(r)\right)\right)$ separates $E$, i.e. $U_{0} \supset B^{n}\left(x, r_{1}\right)$ and $\bar{U}(x, f, r) \subset \bar{B}^{n}\left(x, L^{*}(r)\right)$, we have

$$
\operatorname{cap} E \leqq \operatorname{cap}\left(B^{n}\left(x, r_{1}\right), \bar{B}^{n}\left(x, L^{*}(r)\right)\right)=\frac{\omega_{n-1}}{\left(\log \left(r_{1} / L^{*}(r)\right)\right)^{n-1}} .
$$

The inequality $(6.2)$ yields

$$
r \leqq C_{1} L^{*}(r)^{\alpha^{1 /(n-1}}
$$

where $C_{1}=r_{0} / r_{1}^{\alpha^{1(n-1)}}$. By the metric characterization of quasiregular mappings [7, Theorem 4.6 , p. 19]

$$
\frac{L^{*}(r)}{l^{*}(r)} \leqq C_{2}
$$

for $0<r \leqq r_{2}$. But

$$
\frac{L^{*}(r)}{|x-y|} \leqq \frac{L^{*}(r)}{l^{*}(r)} \leqq C_{2}
$$


which implies, by (6.3),

$$
|f(x)-f(y)|=r \leqq C_{1} C_{2}^{\alpha^{1 /(n-1)}} \mid x-y^{x^{1(n-1)}} .
$$

Since this holds for every $y \in U\left(x, f, r_{2}\right)$ and $\alpha^{1 /(n-1)}>1, f$ is differentiable at $x$ and $f^{\prime}(x)=0$. The theorem follows.

6.4. Remark. The inequality $K_{I}(f)<i(x, f)$ in Theorem 6.1 is best possible, i.e. there exists a quasiregular mapping $f$ such that $K_{I}(f)=$ $i(x, f)$ and $f$ is not differentiable at $x$. The winding mapping $f: R^{3} \rightarrow R^{3}$, $(r, \varphi, z) \mapsto(r, 2 \varphi, z)$ in cylindral coordinates, gives an example since $i(0, f)=K_{I}(f)=2$ and it is not differentiable at 0 . Similar examples exist in all dimensions $n \geqq 2$. A question arises: Does there exist any quasiregular mapping $f: G \rightarrow R^{n}, n \geqq 3$, such that $K_{I}(f)<i(x, f)$ for some $x \in G$ ? However, the example of Rickman [11] shows that $i(x, f)$ has no upper bound in terms of $K_{I}(f)$.

6.5. Corollary. Suppose that $f: G \rightarrow R^{n}$ is a non-constant quasiregular mapping and $\alpha:[a, b] \rightarrow B_{f}$ a rectifiable curve. Then

$$
K_{I}(f) \geqq \inf _{x \in|\alpha|} i(x, f) .
$$

The bound is best possible.

Proof. Integrate $f^{\prime}(x)$ along $|\alpha|$ and use Theorem 6.1 and Remark 6.4.

6.6. Corollary. If $f: G \rightarrow R^{n}$ is a non-constant quasiregular mapping and $\alpha:[a, b] \rightarrow B_{f}$ is a rectifiable curve, then $K_{J}(f) \geqq 2$. The bound is best possible.

6.7. The author conjectures that Corollaries 6.5 and 6.6 hold if $\alpha$ is any continuum in $B_{f}$. Anyway, we obtain the following weaker result.

6.8. Theorem. Suppose that $f: G \rightarrow R^{n}$ is a non-constant quasiregular mapping and $F$ is a compact set in $B_{f}$ such that $1_{p}(f F)>0$. Then

$$
\frac{K_{I}(f)}{\inf _{x \in F} i(x, f)}>\left(\frac{p}{n}\right)^{n-1} .
$$
$\partial f(G)$

Proof. Following $\left[7,4.1\right.$, p. 17] we set for $x \in G_{r}$ and $0<r<d(f(x)$,

$$
\begin{aligned}
& L^{*}(x, f, r)=\sup x-z_{i}, \\
& l^{*}(x, f, r)=\inf \quad x-z
\end{aligned}
$$


over $z \in \partial U(x, f, r)$ and for $0<r<d(x, \partial G)$

$$
L(x, f, r)=\sup |f(x)-f(y)|
$$

over $|x-y|=r$. By $[7$, Theorem 4.6, p. 19] there exists $a>0$ such that

$$
H^{*}(x, f)=\varlimsup_{r \rightarrow 0} \frac{L^{*}(x, f, r)}{l^{*}(x, f, r)}<a
$$

for all $x \in F$. By $\left[7\right.$, Lemma 4.8, p. 20] we may find an interval $\left(0, r_{1}\right)$ such that the mappirg $(x, r) \mapsto L^{*}(x, f, r)$ is lower semicontinuous and the mapping $(x, r) \mapsto l^{*}(x, f, r)$ is continuous on $F \times\left(0, r_{1}\right)$. Hence for $1 / i<r_{1}$ the sets

$$
C_{i}=\left\{x \in F \mid L^{*}(x, f, r) / l^{*}(x, f, r) \leqq a \text { for all } 0<r<1 / i\right\}
$$

are compact. Furthermore $\mathrm{U} C_{i}=F$, thus it is possible to fix $i$ such that $A_{p}\left(f C_{i}\right)>0$. Since the mapping $x \mapsto i(x, f)$ is upper semicontinuous, the sets $C_{i j}=\left\{x \in C_{i}: i(x, f)=j\right\}, j=2,3, \ldots$, are Borel sets. Fix $j$ such that $A_{p}\left(f C_{i j}\right)>0$. Pick $x_{0} \in C_{i j}$ and $r_{0}$ with the properties (i) $0<r_{0}<1 / i$, (ii) $C_{0}=U\left(x_{0}, f, r_{0}\right)$ is a normal neighbourhood of $x_{0}$. and (iii) $A_{p}\left(f\left(C_{i j} \cap U\right)\right)>0$ for all $U=U\left(x_{0}, f, r\right), 0<r \leqq r_{0}$. Choose $r_{0}^{\prime}>0$ such that $\bar{B}^{n}\left(x_{0}, 2 r_{0}^{\prime}\right) \subset U_{0}$ and then $r_{0}^{\prime \prime}>0$ such that $\bar{U}\left(x_{0}, f, r_{0}^{\prime \prime}\right) \subset B^{n}\left(x_{0}, r_{0}^{\prime}\right)$.

$\mathrm{By}$ the continuity of $f$, the function

$$
\sigma(r)=\sup _{x \in F} L(x, f, r)
$$

$r \in(0, d(F, \partial G))$, has the property $\sigma(r) \rightarrow 0$ as $r \rightarrow 0$. Suppose that $t_{1} \in(0, d(F, \partial G))$ is such that $r \in\left(0, t_{1}\right]$ implies

$$
\sigma(r)<d\left(f S^{n-1}\left(x_{0}, r_{0}^{\prime}\right), B^{n}\left(f\left(x_{0}\right), r_{0}^{\prime \prime}\right)\right)>0 .
$$

Assume now that the condition

$$
\frac{K_{I}(f)}{\inf _{x \in F} i(x, f)} \leqq\left(\frac{p}{n}\right)^{n-1}
$$

holds. Denote $F_{1}=C_{i j} \cap U\left(x_{0}, f, r_{0}^{\prime \prime}\right)$. Te show that $A_{p}\left(f F_{1}\right)=0$. Let $t>0$ and $\varepsilon>0$. By [7, Theorem 8.3, p. 38] $m\left(B_{f}\right)=0$, and so $A_{n}\left(F_{1}\right)=0$. Hence it is possible to cover $F_{1}$ by balls $B^{n}\left(x_{k}, r_{k}\right)$. $k=1,2, \ldots$, such that (1) $x_{k} \in F_{1}$, (2) $r_{k}<t_{1}, \quad$ (3) $\sigma\left(r_{k}\right)<t / 2$, and (4) $\sum r_{k}^{n}<\varepsilon$. Fix $k$ and define $E_{k}=\left(U_{0}, \bar{C}\left(x_{k}, f, L_{k}\right)\right)$ where $L_{k}=$ $L\left(x_{k}, f, r_{k}\right)$. Then $E_{k}$ is a condenser in $G$ since, by $(2), \bar{U}\left(x_{k}, f, L_{k}\right) \subset$ $B^{n}\left(x_{0}, r_{0}^{\prime}\right) \subset U_{0}$. Because $i\left(x_{0}, f\right)=i\left(x_{k}, f\right)$, the set $U\left(x_{k}, f, L_{k}\right)$ is a normal neighbourhood of $x_{k}$. Thus Lemmas 3.7 and 3.4 yield 
$M\left(f, \bar{C}\left(x_{k}, f, L_{k}\right)\right)=\mu\left(f, U\left(x_{k}, f, L_{k}\right)\right)=i\left(x_{k}, f\right)=i\left(x_{0}, f\right)=N\left(f, L_{0}\right)$. Corollary 5.13 and (6.10) imply

$$
\operatorname{cap} f E_{k} \leqq \frac{K_{I}(f)}{i\left(x_{0}, f\right)} \operatorname{cap} E_{k} \leqq\left(\frac{p}{n}\right)^{n-1} \text { cap } E_{k}
$$

On the other hand

$$
\operatorname{cap} f E_{k} \geqq \frac{\omega_{n-1}}{\left(\log \left(2 r_{0} / L_{k}\right)\right)^{n-1}}
$$

since the condenser $f E_{k}$ separates the condenser $\left(B^{n}\left(f\left(x_{k}\right), 2 r_{0}\right)\right.$, $\left.\bar{B}^{n}\left(f\left(x_{k}\right), L_{k}\right)\right)$ and

$$
\operatorname{cap} E_{k} \leqq \frac{\omega_{n-1}}{\left(\log \left(r_{0}^{\prime} / L_{k}^{*}\right)\right)^{n-1}}
$$

since $\left(B^{n}\left(x_{k}, r_{0}^{\prime}\right), \bar{B}^{n}\left(x_{k}, L_{k}^{*}\right)\right), \quad L_{k}^{*}=L^{*}\left(x_{k}, f, L_{k}\right)$, separates $E_{k}$. But $U\left(x_{k}, f, L_{k}\right)$ is a normal neighbourhood of $x_{k}$, hence we conclude from $\left[7\right.$, Lemma 4.3, p. 18] that $l_{k}^{*}=l^{*}\left(x_{k}, f, L_{k}\right)=r_{k}$. Thus the above three inequalitics yield

$$
L_{k} \leqq \frac{2 r_{0}}{r_{0}^{\prime n / p}} L_{k}^{* n^{\prime} p} \leqq \frac{2 r_{0} a^{n / p}}{r_{0}^{\prime n / p}} l_{k}^{* n / p}=b i_{k}^{n p} .
$$

The set $f F_{1}$ is covered by $\cup f B^{n}\left(x_{k}, r_{k}\right)$ and, by (3). $d\left(f B^{n}\left(x_{k}, r_{k}\right)\right)<t$. hence we obtain from (6.11) and (4)

$$
A_{p}^{t}\left(f F_{1}\right) \leqq \sum d\left(f B^{n}\left(x_{k}, r_{k}\right)\right)^{p} \leqq 2^{p} \sum L_{k}^{p} \leqq 2^{p} b^{p} \sum r_{k}^{n}<2^{p} b^{p} \varepsilon .
$$

Thus $A_{F}\left(f F_{1}\right)=0$, a contradiction of (iii). The theorem follows.

6.12. Corollary. Suppose that $f: G \rightarrow R^{n}$ is a non-constant quasiregular mapping and $F \subset B_{f}$ is a continuum. Then

$$
\frac{K_{I}(f)}{\inf _{x \in F} i(x, f)}>n^{1-n} .
$$

Proof. Since $f$ is light, $\Lambda_{1}(f F)>0$, hence the corollary follows from Theorem 6.8.

6.13. Definition. A non-constant quasiregular mapping is said to be of minimal multiplicity $q$ if $B_{f} \neq \varnothing$ and $i(x, f) \geqq q$ for all $x \in B_{f}$.

6.14. Theorem. Suppose that $f: G \rightarrow R^{3}$ is of minimal multiplicity q. Then $K_{I}(f)>q / 9$. 
Proof. By [12] $\operatorname{dim} B_{f}=1$, hence there is a continuum $F \subset B_{f}$ ([6, II 4, p. 20]). Thus the theorem follows from Corollary 6.12 .

6.15. Remark. We have been able to prove that $K_{I}(f)>q(n /(n-2))^{1-n}$ for every quasiregular mapping $f: G \rightarrow R^{n}$ of minimal multiplicity $q$ in any dimension $n \geqq 3$. The authors of $[7]$ intend to return to related questions in a later paper.

6.16. Remark. Theorem 6.14 gives a rather good asymptotic estimate for the growth of $K_{I}(f)$ in terms of $q$. In fact, the winding mapping $f: R^{3} \rightarrow R^{3},(r, \varphi, z) \mapsto(r, q \varphi, z)$ in cylindrical coordinates. is of minimal multiplicity $q$ and $K_{I}(f)=q$. Hence

$$
q \geqq \inf _{f \in W_{q}} K_{I}(f) \geqq q / 9
$$

where $W_{q}$ is the family of all quasiregular mappings into $R^{3}$ of minimal multiplicity $q$. If the conjecture in 6.7 is true, then

$$
\inf _{f \in W_{q}} K_{I}(f)=q,
$$

in particular, there would not exist a non-constant quasiregular mapping $f: G \rightarrow R^{3}$ with non-empty $B_{f}$ such that $K_{I}(f)<2$.

Univesity of Helsinki

Helsinki, Finland 


\section{References}

1. Černavski, A. V. (Чернавсний, А. В.): Конечногратные оттрытые отображения многообразпй. - Mat. Sbornik 65 (1964), 357-369.

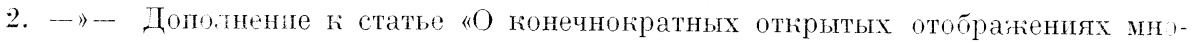
гообразий”. - Mat. Sbornik 66 (1965), 471-472.

3. Church, P. T. and Hemmingses, E.: Light open maps on $n$-manifolds. - Duke Math. J. 27 (1960), 527-536.

4. Gemring, F. W.: Symmetrization of rings in space. - Trans. Amər. Math. Soc. 101 (1961), 499-519.

5. - - - Rings and quasiconformal mappings in space. - Trans. Amэr. Math. Soc. 103 (1962), 353-393.

6. Hurewicz, W. and Walladax, H.: Dimension theory. - Princeton University Press, 1941.

7. Martio, O., Rickman, S. and Vätsälä, J.: Definitions for quasiregular mappings. . Ann. Acad. Sci. Fenn. A I 448 (1969), 1-40.

8. Martio, O.: Behaviour of capacities under quasiregular mappings. - Proceedingof the Roumanian-Finnish sominar on Teichmüller spacs and quasiconformal mappings (to appear).

9. Rešetnjak, J. G. (Решетнян. IO. Г.): IІространственные отображепия с ограниченным псканением. - Sibirsk. Mat. Z̆. 8 (1967), 629-65̃8.

10. - - Об успови огранцченности пндекса дтя отображений с ограниченным псканеннем. - Sibirsk. Mat. Ž. 9 (1968), 368-374.

11. Rickman, S.: Quasiregular mappings. - Proceedings of the Roumanian-Finnish seminar on Teichmüller spaces and quasiconformal mappings (to appear).

12. Твонтме̌к, J. J. (Трохимчук, Ю. Ю.): О непрерывных отобрағіених областеї әвк.пи,това прострапетва. - Ukrain. Мat. Z̆. 16 (1964). 196-211.

13. VÄıs̈̈LÄ, J.: Discrete open mappings on manifolds. - Ann. Acad. Sci. Fenn. A I $392(1966), 1-10$.

14. - - - Lectures on $n$-dimensional quasiconformal mappings. - Tan Nostrand (to appear).

15. Whyburs, C. T.: Analytic topology. - Amer. Math. Soc. Colloquium Publications, 1942. 